\title{
First Demonstration of All-Optical QPSK Signal Regeneration in a Novel Multi-Format Phase Sensitive Amplifier
}

\author{
Joseph Kakande ${ }^{(1)}$, Adonis Bogris ${ }^{(2)}$, Radan Slavík ${ }^{(1)}$, Francesca Parmigiani ${ }^{(1)}$, Dimitris Syvridis ${ }^{(2)}$, \\ Periklis Petropoulos ${ }^{(1)}$, and David J. Richardson ${ }^{(1)}$ \\ (1) Optoelectronics Research Centre, University of Southampton, Southampton, SO17 1BJ, UK \\ Tel.:+44-2380594524, Fax+44-2380593142, e-mail:jkk@orc.soton.ac.uk \\ (2) Department of Informatics and Telecommunications, National and Kapodistrian University of \\ Athens, Panepistimiopolis, Ilissia, 15784, Athens, Greece
}

Abstract We propose a novel black-box optical phase sensitive amplifier (PSA) configuration and describe its application to the regeneration of multi-level phase encoded signals. The concept is demonstrated with a 10 Gbaud quadrature phase shift keyed (QPSK) input.

\section{Introduction}

The future of optical fiber communications will be dictated by the need for long reach, high capacity and energy efficient technologies. Transitioning to spectrally efficient modulation formats such as QPSK provides significant capacity gains in long haul optical links. Fully coherent optical signal detection combined with high speed analog-to-digital conversion allows signal processing in the electronic domain, providing capabilities such as compensation for chromatic and polarization mode dispersion, as well as for some of the accumulated nonlinear phase noise which is the dominant limitation in extending coherent transmission spans ${ }^{1}$. However, the power consumption as well as the significant computing overhead associated with the aforementioned electronic functions ${ }^{2}$ means that a combination of optical signal processing with optical dispersion compensation may still prove competitive for long haul transmission, particularly as signalling rates continue to rise.

A long term goal of research into alloptical signal regeneration has been to identify techniques capable of processing advanced formats with multiple levels in phase or/and amplitude. One proposed scheme utilises a pair of conjugated signal-idler channels transmitted along the link and combined in a nondegenerate $\mathrm{PSA}^{3}$; however this wastes valuable transmission bandwidth, and requires impractically low levels of residual dispersion post-compensation. Other proposals to all- optically regenerate QPSK have focussed on scaling schemes intended for use with binary level signals, including an indirect approach utilising format conversion to on-off-keying (OOK), OOK regeneration, and OOK to DQPSK conversion ${ }^{4}$ as well as a more direct technique using two parallel binary phase shift keying (BPSK) regenerators ${ }^{5}$. Such schemes are significantly complicated by the requirement to fully length-match and stabilise multiple optical paths, as well as a component count that increases appreciably with the density of the modulation format, potentially offsetting some of the economic benefits of the spectrally efficient formats. To date, there have been no experimental demonstrations.

We propose a novel concept using inline phase sensitive fiber optical parametric amplifiers to provide the direct all-optical regeneration of arbitrary multilevel phase encoded signals. We use it in the first demonstration to our knowledge of black-box alloptical QPSK phase regeneration (as in Fig.1).

\section{Concept}

Multi-level phase regeneration requires a staircase phase transfer function. For an M-level optical PSK signal, we propose to achieve this by interfering it with a conjugated $(\mathrm{M}-1)^{\text {th }}$ phase harmonic. A semi-analytical way to understand this is as follows. Given QPSK signals for which $\mathrm{M}=4$, a simple way to express this is; $A \cdot \exp \left(i \cdot \phi_{\text {out }}\right)=\exp (i \cdot \phi)+m \cdot \exp (-i \cdot 3 \phi)$

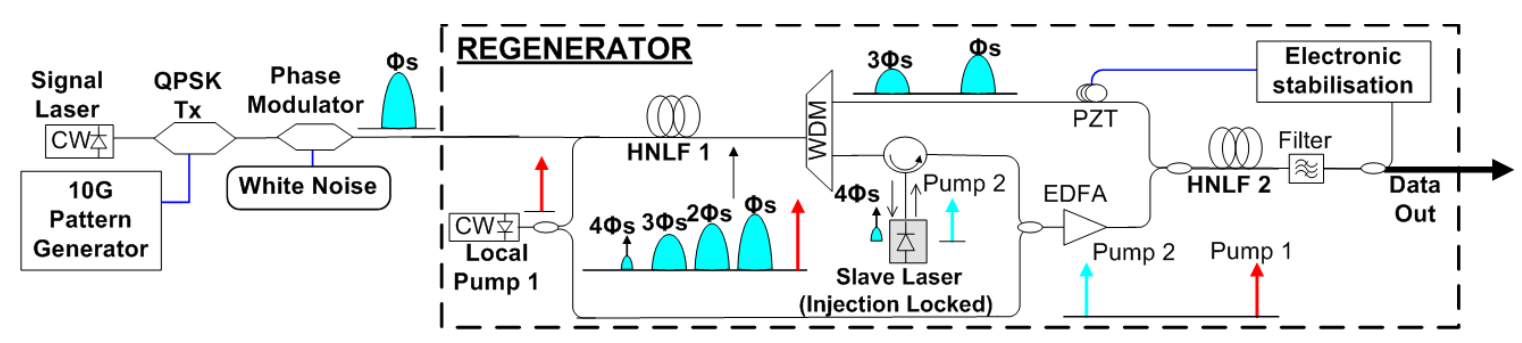

Fig. 1: Experimental setup, PZT - piezo fiber stretcher, HNLF - highly nonlinear fiber, CW - continuous wave, QPSK Tx QPSK transmitter. 

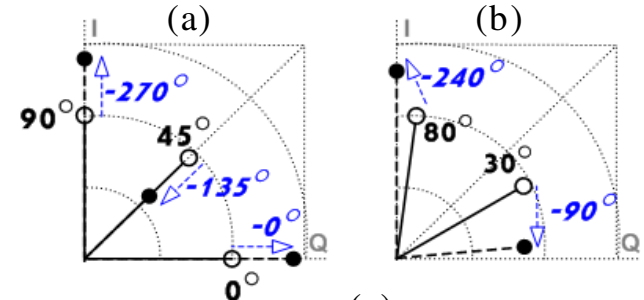

(c)

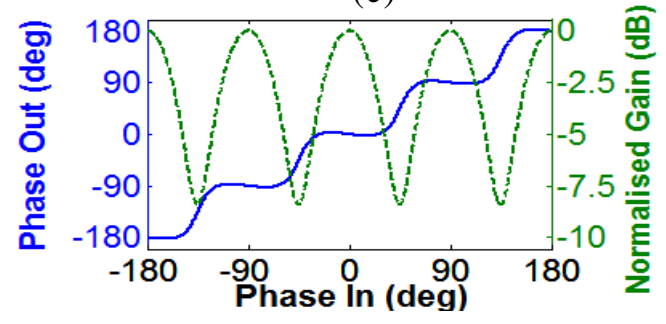

Fig. 2: (a) Illustration of how amplification of $I$ and $Q$ quadratures is achieved while the $45^{\circ}$ component is deamplified. White circles, input $\phi$, dotted vector $-3 \phi$, black circles, output (b) Example with symbols at $30^{\circ}$ and $80^{\circ}$ (c) Complete semi-analytical transfer function showing phase and amplitude response $(m=0.4)$.

where $\phi$ is the input signal modulation, $\phi_{\text {out }}$ is the output modulation (regenerated), $A$ is a phase-to-amplitude conversion term and $m$ is a coefficient to optimise the phase regeneration.

Fig. 2(a) and (b) illustrate in a simple fashion how this provides phase regeneration. For symbols aligned to the principal axes of $0^{\circ}$ and $90^{\circ}$ (I and $\left.\mathrm{Q}\right), \phi$ and $-3 \phi$ are exactly in phase with each other, and their combination leads to constructive interference as shown in Fig. 2(a). However, for symbols aligned at $45^{\circ}, \phi$ and $-3 \phi$ are $180^{\circ}$ out of phase leading to destructive interference. Fig 2(b) shows an example of the phase re-alignment for two sample points with input phases of $30^{\circ}$ and $80^{\circ}$. The value of $m$ optimises the overall phase transfer characteristics; for a $\pi / 2$ step period (i.e. a QPSK signal) it should be approximately 0.35-0.5 (in amplitude, not power). The complete transfer function is shown in Fig. 2(c). Detailed numerical simulations have been carried out verifying this analytical approach.

To practically achieve multilevel phase regeneration, we utilise a two step process. First, the QPSK signal is mixed with a pump to generate a four wave mixing (FWM) comb, including the required $3 \phi$ (referred to from now on as the harmonic), see Fig. 3. The signal and

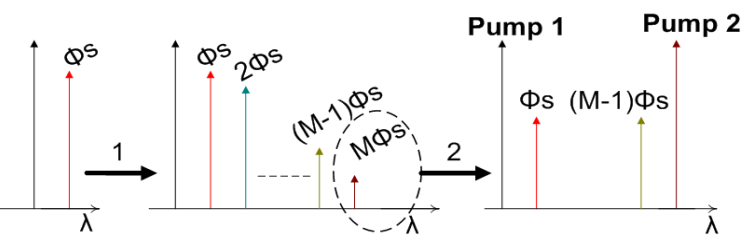

Fig. 3: Regeneration in two steps - 1) Nonlinear generation of phase harmonics via four wave mixing (FWM) followed by pump recovery, 2) Parametric phase sensitive gain harmonic are then combined inside a dual pump non-degenerate PSA where the coherent addition occurs. Of the two required pumps, the first is derived from the free running laser originally used in the comb generation stage, and the second by injection locking the $4 \phi$ (modulation stripped) wave to a semiconductor laser, satisfying the phase locking requirement. The relative powers of the signal and harmonic need to be optimised to take the amplifier power gain $G$ into account; as such a signal-harmonic offset of $m_{\text {eff }}$ is used, where $m_{\text {eff }}=m^{*} \operatorname{sqrt}(1-1 / G)$.

Reconfiguring the regenerator to an alternate modulation format such as 8-PSK can be achieved simply by generating a broader FWM comb (by increasing the signal and pump powers), passively selecting the desired harmonic and tuning the injection locked laser to the corresponding frequency.

\section{Experimental Setup}

A CW wave at $1555.7 \mathrm{~nm}$ was modulated with a pseudo-random binary sequence (PRBS) to generate a 10 Gbaud QPSK signal. To emulate the effects of nonlinear phase noise, the signal was coupled through a $\mathrm{LiNbO}_{3}$ phase modulator driven by variable power levels of electrical white noise spanning up to $8 \mathrm{GHz}$. The signal was amplified to $22 \mathrm{dBm}$ and combined in HNLF 1 (OFS) with a $14 \mathrm{dBm}$ portion of pump 1 at $1557.5 \mathrm{~nm}$ to generate the FWM comb as shown in Fig. 4. HNLF1 was $500 \mathrm{~m}$ long with nonlinear coefficient $10.7 \mathrm{NW} / \mathrm{km}$, zero dispersion wavelength (ZDW) $1544 \mathrm{~nm}$ and dispersion slope (DS) $0.029 \mathrm{ps} / \mathrm{nm}^{2} / \mathrm{km}$. The $4 \phi$ term at $1551.2 \mathrm{~nm}$ was de-multiplexed from the comb and injected into a semiconductor laser ${ }^{6}$, providing pump 2. The rest of the comb was passively filtered out leaving the signal and harmonic at $1552.7 \mathrm{~nm}$. These were combined with the pumps in HNLF 2 (OFS), with a total pump power of $24 \mathrm{dBm}$. This fiber had a length of $300 \mathrm{~m}$, nonlinear coefficient $11.6 \mathrm{~N} / \mathrm{km}$, ZDW $1553 \mathrm{~nm}$ and DS $0.018 \mathrm{ps} / \mathrm{nm}^{2} / \mathrm{km}$. It also had a strain gradient to increase its stimulated Brillouin scattering (SBS) threshold. Any slow relative phase drifts at the PSA input were eliminated by monitoring the signal power at the PSA output and controlling a PZT. The signal was then assessed using a self-homodyne constellation analyser. The sampling rate of the constellation analyser was the limiting factor in the choice of signal baud rate for the experiment.

\section{Results and discussion}

The PSA input is shown in Fig. 5(a). The input signal-to-harmonic power offset was $6 \mathrm{~dB}$. The phase sensitive extinction was measured at around $7 \mathrm{~dB}$ as shown in Fig. 5(b). 


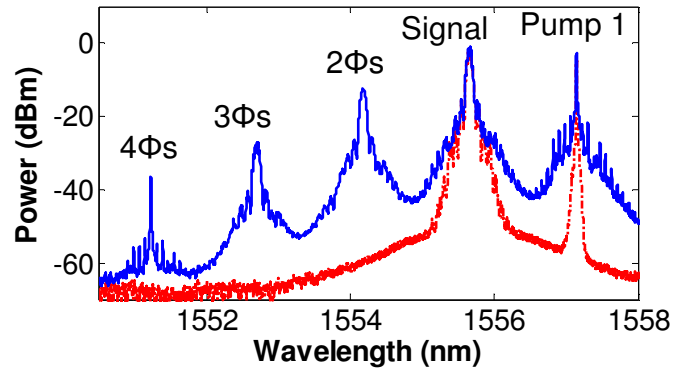

Fig. 4: FWM comb generation stage input (dotted) and output(solid)
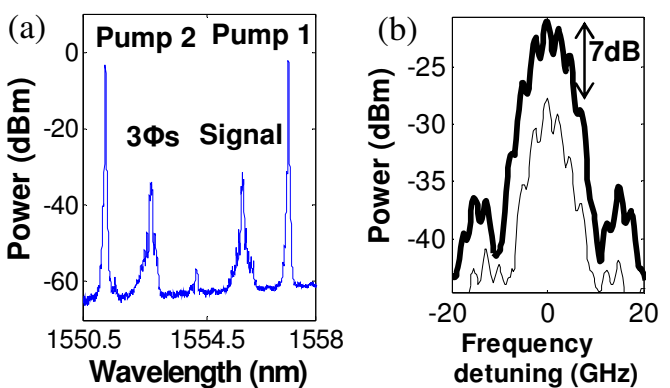

Fig. 5: (a) HNLF2 input, (b) Signal spectrum at HNLF2 output, thick line (top) is PS maximum, thin line is PS minimum

The constellation diagrams in Fig. 6 show the regeneration for three added phase noise levels. In the absence of any added noise, there was a slight degradation; see Fig. 6(a), (b). This stems primarily from the amplification and filtering of the signal within the regenerator, as well as ASE added by the EDFA amplified pumps. Future system iterations should remedy this degradation.

Absolute phase deviations of up to $60^{\circ}$ per symbol were squeezed down to about $30^{\circ}$; see Fig. 6(c), (d). The regenerator was able to squeeze even larger phase fluctuations (6(e), (f)) but this was accompanied by phase-tointensity conversion. It is possible to suppress this by saturating the PSA as shown by our numerical simulations. However, for phase only formats such as QPSK, amplitude fluctuations impose little penalty, and therefore this is not crucial.

The level of squeezing illustrates one of the key benefits of PSA regenerators: assuming they are placed before a differential optical receiver, they have the potential to significantly reduce the BER for severely degraded signals. This is because phase deviations are magnified by up to a factor of 2 during differential detection and therefore absolute deviations over $\pm 22.5^{\circ}$ for DQPSK can cause errors when differentially decoded, but these can be eliminated by this regenerator.

It is worth pointing out that the regeneration of formats containing signalling in both amplitude and phase such as square 16-QAM can be regenerated by placing two such QPSK
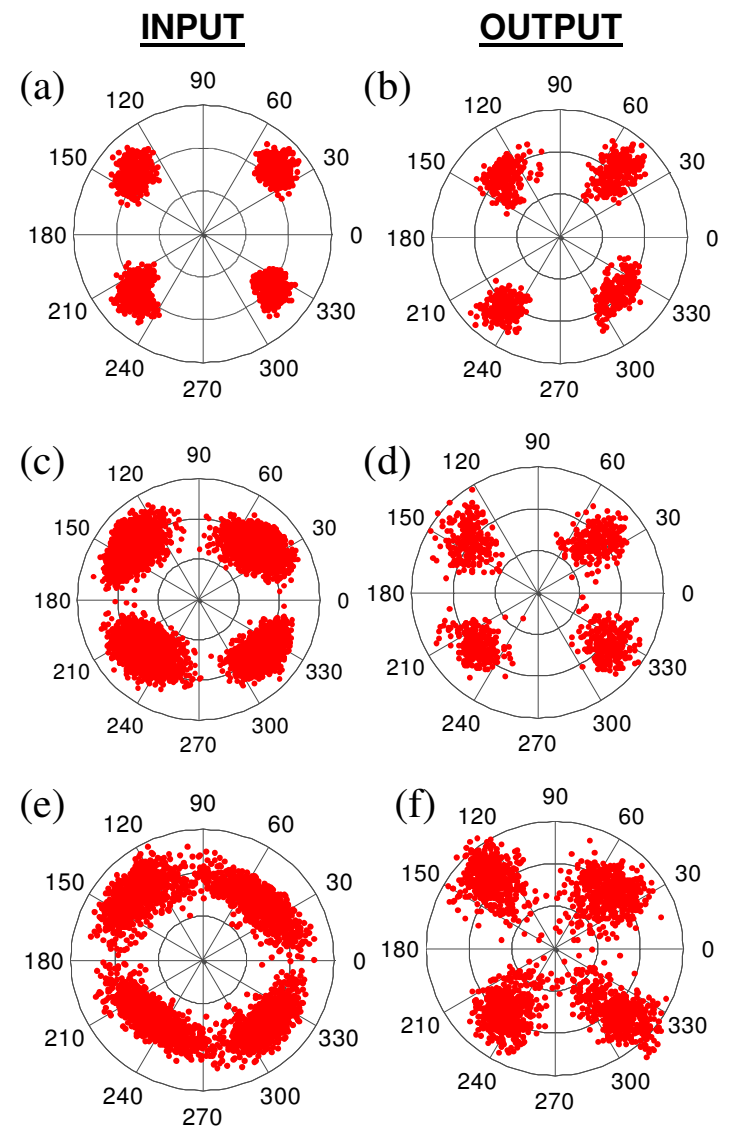

Fig. 6: Signal constellations, (a),(c) and (e) regenerator input, (b), (d) and (f) regenerator output respectively

regenerators in parallel provided the incoming signal is first optically de-multiplexed into two QPSK channels. As such this device truly redefines the possibilities of all optical signal processing.

\section{Conclusions}

In conclusion, we have proposed a novel optical signal processing function, enabling the first experimental demonstration of phase regeneration of a multilevel PSK signal. This has been shown with a 10 Gbaud QPSK signal, reducing white noise phase deviations by a factor of at least 2.

\section{Acknowledgements}

We would like to thank OFS Fitel Denmark for providing the HNLFs used. This research has received funding from the European Communities Seventh Framework Programme FP/2007-2013 under grant agreements 224547 (PHASORS) and 216863 (BONE).

\section{References}

1 E. Ip et. al., Opt. Express 16, 753-791 (2008).

2 K. Roberts et. al., J. Lightwave Technol. 27, 3546-3559 (2009).

3 M. Vasilyev, Opt. Exp., 13, 7563 (2005).

4 M. Matsumoto, Opt. Express 18, 10-24 (2010).

5 Z. Zheng et. al., Optics Communications 281, 2755-2759 (2008).

6 R. Weerasuriya et al., OFC'2010, OWT6. 